\title{
Editorial: Diagnosis of cell-mediated immunity in ocular disease
}

It is generally agreed that the lymphoid cells involved in immunity and its disorders are of two distinct types, known respectively as T- and B-lymphocytes. T-lymphocytes are derived from lymphoid cells in the bone marrow which migrate to the thymus during embryogenesis and early infancy and there develop the ability to respond to an antigen. Such thymus-derived cells are the predominant lymphocytes in the peripheral blood, give rise to well-defined areas in the lymph nodes and the spleen, and are responsible for delayed hypersensitivity phenomena and transplantation reactions. The second type of lymphoid cell, known as the B-lymphocyte, similarly arises in the bone marrow but differentiates under the influence of the bursa of Fabricius (in birds), or its analogue in mammals, and ultimately settles in the lymphoid organs in areas quite distinct from those occupied by T-lymphocytes. Under appropriate antigenic stimuli these cells transform into plasma cells and secrete the various classes of immunoglobulin.

Immunological tests designed to assess the functional state of both types of lymphocyte are of relevance, not only in acute and chronic ocular infections, but also in allergy, autoimmunity, and neoplasia of the eye. Antibody-mediated immune reactions have long been studied by several sensitive serological procedures, such as precipitation, haemagglutination, and complement-fixation tests. Delayed hypersensitivity, on the other hand, has been demonstrated until recently only by means of delayed skin reactions (e.g. the Mantoux test). In vivo tests of this sort necessitate the re-introduction of antigen into a sensitized individual, and this is liable to produce adverse effects, such as an acute allergic reaction, re-activation of a dormant lesion, or accentuation of the delayed hypersensitivity state. Moreover, these skin reactions are difficult to quantitate and may be suppressed in patients receiving anti-inflammatory drugs. To circumvent these difficulties several sensitive in vitro tests have been introduced which are easily controlled and quantitated. T-lymphocytes obtained from sensitized donors can be shown to react in vitro with the appropriate antigen not only by undergoing a blastic transformation and mitotic division, but also by acquiring the ability to kill the target cells and secrete biologically active substances known collectively as "lymphokines". Lymphokines include substances such as migration inhibition factor (MIF), macrophage aggregation factor (MAF), chemotactic factor, mitogenic factor, lymphotoxin, skin reactive factor, transfer factor, etc. One of the tests which utilize these properties to measure $\mathrm{T}$ - cell reactivity (i.e. delayed hypersensitivity) is the leucocyte migration inhibition test. (Søborg and Bendixen, 1967).

Normal human leucocytes, when packed in capillary tubes and placed in small tissue culture chambers, tend after some time to migrate out and to form a "fan" of cells at the bottom of the chamber. If specific antigen is present in the medium, MIF is produced by the activated T-lymphocytes and migration is inhibited.

Patients' leucocytes cultured in the presence of the suspected antigen will show a similar inhibition of movement. By comparing the size of the "cellular fan" obtained after I 6 to 24 hrs' incubation in the presence of the antigen with that of the control chamber lacking that antigen, the result is expressed either as percentage inhibition or as migration index.

In comparison with other tests for cellular immunity, such as the lymphocyte trans- 
formation test, the colony inhibition test, and the cell-mediated cytotoxicity test, this test is relatively simple to perform and correlates well with the delayed skin hypersensitivity response (Roseberg and David, I97I). A marked inhibition of leucocyte migration has been observed in microbial hypersensitivity, organ-specific diseases, and certain drug allergies. Only recently it has been shown that this test is of some relevance in Sjögren's disease (Berry, Bacon, and Davis, 1970), Wegener's granulomatosis (Shillitoe, Lehner, Lessof, and Harrison, I974), chronic viral keratitis, and choroidoretinitis (Leopold, 1973). It is of interest that Hammer now shows in the following article that a positive result is also a feature of sympathetic ophthalmitis.

\section{References}

BERRY, H., BACON, P. A., and DAvis, J. D. (1972) Ann. rheum. Dis., 31, 298

LEOPOLD, I. H. (1973) Amer. F. Ophthal., 76, 619

ROSEBERG, S. A. and DAVID, J. R. (I97I) In "In vitro Methods in Cell-mediated Immunity", ed. B. R.

Bloom and P. R. Glade, p. 297. Academic Press, New York.

SHILLITOE, E. J., LEHNER, T., LESSOF, M. H., and HARRISON, D. F. N. (1974) Lancet, 1, 28 I

SøBORG, M., and BENDIXEN, G. (1967) Acta med. scand., 181, 247 